\title{
La educación física en las organizaciones educativas, un estudio de caso en centros educativos públicos de secundaria en el circuito 10 en la Dirección Regional de Educación de San José, Costa Rica
}

\author{
Physical Education in educational organizations, an \\ analysis from the educational management: a case study \\ in public schools from the management of high school \\ education in the $10^{\text {th }}$ Circuit of the Regional Educational \\ Directions in San José, Costa Rica
}

\author{
Esteban Pereira Hidalgo ${ }^{1}$ \\ Comité Cantonal de Deportes y Recreación de Montes de Oca (CCDRMO) \\ estecomite@gmail.com
}

\begin{abstract}
Recibido 21 noviembre 2011 * Aceptado 24 noviembre 2011 * Corregido 02 diciembre 2011
\end{abstract}
Resumen. El presente artículo refiere a un análisis de gestión que podría darse entre las instituciones educativas públicas de secundaria del circuito 10 de la Dirección Regional de San José y el Comité Cantonal de Deportes y Recreación de Montes de Oca, en procura del mejoramiento de la Educación Física de dichos centros. Para esta investigación, se consideró como objetivos la descripción del proceso administrativo ejecutado por las direcciones de los centros educativos, específicamente en el área de la educación física; la determinación del proceso de coordinación existente entre los centros educativos en estudio y la organización municipal; la identificación del conocimiento que tienen los actuales profesionales en la administración de la educación en los centros educativos públicos con respecto de las reformas que involucran a las organizaciones educativas y finalmente, el establecimiento de un análisis comparativo entre las instituciones, en función del impacto que la gestión ha tenido en el área de la Educación Física, en torno a la reforma al artículo 170 del Código Municipal. El sustento teórico que cimienta este estudio cualitativo exploratorio, se centra en la posición de la Organización de las Naciones Unidas (ONU) con respecto del deterioro que ha sufrido la educación en los aspectos de infraestructura, recursos materiales, pero sobre todo en el desarrollo físico, social, estético y moral de la población estudiantil. Para ello, se realizaron entrevistas semiestructuradas a los directores y al cuerpo docente de Educación Física de los centros educativos, cuyos resultados muestran que las direcciones consultadas llevan a cabo las acciones necesarias para mejorar en el plano institucional en lo que a deporte y recreación se refiere, pero ninguna ha coordinado planes o

\footnotetext{
${ }^{1}$ Magíster en Ciencias de la Educación con énfasis en Administración de la Educación de la Universidad de Costa Rica. Bachiller en la enseñanza de la Educación Física y ostenta un grado de especialista en alto rendimiento deportivo. Ha dedicado gran parte de su carrera a la promoción de vínculos entre las organizaciones educativas y comunales a través del deporte y la recreación desde el Comité de Deportes y Recreación de Montes de Oca, donde se desempeña como Director Deportivo.
}

Número publicado el 20 de diciembre de 2011 URL: http://revistadigital.eae.fcs.ucr.ac.crl 
Revista Gestión de la Educación, Vol.1, N², pp.107-143, ISSN 2215-2288, julio-diciembre, 2011

proyectos en conjunto con la administración municipal, y que en la mayoría de los casos, no se toma en cuenta al personal docente para analizar, desde un nivel operativo, las principales necesidades de la organización en el plano deportivo y recreativo. Esta acción es fundamental en el entendido de que el personal del centro educativo se puede convertir en un recurso relevante en el cumplimiento de las metas y objetivos de la organización.

Palabras clave. Educación, administración de la educación, coordinación, gestión de la educación, Educación Física.

Abstract. This article refers to a research that studies management that could occur in public educational institutions of high school education in the $10^{\text {th }}$ Circuit of the Regional Educational Direction in San José and the County Committee of Sports and Recreation of Montes de Oca, in order to improve Physical Education in schools. For this research the following objectives are: describe the administrative process of school management in the physical education area, determine the coordination process in schools studied and county organization, identify the knowledge that administrators of public schools have with regard to reforms involving education organization; and finally, establish a comparative analysis of these institutions, depending on the impact that management has in Physical Education field based on the reform of Article 170 of the Municipal Code. The theoretical basis of this research is founded mainly on the United Nations Organization (UN) position corresponding to the decline suffered by education in areas such as: infrastructure, material resources, but especially in the physical, social, aesthetic and moral development of student population. This research was conducted under an exploratory qualitative approach, with semi-structured interviews directed to principals and teachers of Physical Education in schools. The study results show that principals perform necessary actions to improve the institutional level; as far as, sports and recreation. Mean while, evidence taken show that none of the organizations involved included plans or projects with the municipal administration, and in most cases teachers are not taken into account to analyze the main needs organization concerning to sporting and recreational level. This action is fundamental to understand that school staff can be the main resource to promote and reach the organizational goals and objectives.

Key words. Education, educational administration, coordination, education management, Physical Education.

\section{Introducción}

En la actualidad la educación ha dejado de ser un privilegio de pocos, para convertirse en una necesidad de muchos, teniendo en cuenta que cada vez es mayor la demanda de talento humano en todas las áreas de empleo que la sociedad requiere. La educación sigue siendo el pilar de una sociedad desarrollada o en vías de desarrollo, que a medida que aumentan las necesidades productivas de la mayoría de países del mundo se hace necesaria la incorporación de nuevas estrategias y políticas administrativas que

le den a la educación el papel preponderante que requiere para hacerle frente a dichas necesidades.

Número publicado el 20 de diciembre de 2011 URL: http://revistadigital.eae.fcs.ucr.ac.crl 
Revista Gestión de la Educación, Vol.1, №2, pp.107-143, ISSN 2215-2288, julio-diciembre, 2011

Sumado a lo anterior, uno de los grandes desafíos que enfrenta la universalización de la educación es precisamente llegar hasta los niños y jóvenes que no están asistiendo al centro educativo, no por el acceso a estos, sino por su falta de interés y la poca calidad de la educación que reciben una vez dentro del sistema educativo. En muchos países en vías de desarrollo, los sistemas educativos tienen una insuficiencia crónica de recursos y por lo general, no ofrecen una enseñanza de calidad (Organización de las Naciones Unidas [ONU], 2009).

Este factor está estrechamente relacionado con dos situaciones reales que probablemente tengan una gran incidencia en el desarrollo de la educación de la región. Una de ellas, es el inevitable aumento de la población estudiantil que está abarrotando las aulas, y aunque uno de los grandes objetivos del milenio es la alfabetización de toda la población, muchas organizaciones educativas no tienen las condiciones necesarias para albergar a tantos niños, niñas y jóvenes.

La segunda situación, es que a raíz del problema mencionado anteriormente, la calidad del servicio brindado por las organizaciones educativas se ve claramente disminuida, lo cual afecta tanto a la población estudiantil como a los centros educativos que tienen que hacer lo imposible con sus instalaciones para albergar un gran número de estudiantes cada año. Lo anterior redunda en que hay más estudiantes en cada aula, menos espacio para cada estudiante, menor personalización del proceso de enseñanza aprendizaje y un indudable aumento en el nivel de estrés de las y los docentes al frente de los grupos sobrecargados.

En este contexto, la administración de la educación juega un papel preponderante al frente del mejoramiento continuo de los procesos que se siguen en favor de los sistemas educativos, tanto desde dentro de las organizaciones educativas como fuera de ellas. En este caso las y los administradores de la educación deberán tomar su lugar en la creación de estrategias válidas y concretas para lograr la reforma educativa que desde el seno del gobierno central se gesta en procura del mejoramiento de la educación. Al respecto, se considera que "sin lugar a dudas, la labor del director determina, en gran medida, la forma como se concreta en la cotidianeidad y en los procesos de aula la misión formadora de la escuela" (García, Rojas y Campos, 2002, p.184). 
Revista Gestión de la Educación, Vol.1, N², pp.107-143, ISSN 2215-2288, julio-diciembre, 2011

Actualmente, aunque la mayoría de las reformas educativas se hacen o se plantean para contribuir de manera directa en el mejoramiento de las diferentes áreas de las organizaciones educativas públicas del país, no siempre se obtienen los resultados esperados, por el contrario, muchas de las leyes y reformas permanecen en el papel sinque nadie les dé seguimiento. Un caso es la enseñanza de la Educación Física, la cual surgió en la enseñanza hacia los años 1950. Según Pila (2002) su reconocimiento legal se lo dio la reforma educativa de 1970 y hasta ahora, no se le ha dado el realce que merece por su importancia dentro del currículo, en el cual Vargas y Orozco (2003), citando a Molina (2002), consideran que una buena formación de hábitos en la actividad física le será de gran utilidad al educando, conforme entre en edades más maduras.

Hacia finales de 2008 surge convenientemente, una reforma al Código Municipal (Ley 7794) que podría representar una de las más grandes reformas legales a favor de la educación, específicamente de la Educación Física de los centros educativos públicos. Esta reforma que involucra los artículos 164 y 170 de dicho Código, hace referencia a dos organizaciones totalmente desvinculadas entre sí, pero que indirectamente se desarrollan en campos vinculantes como lo son la educación y el deporte. Evidentemente, las primeras son las organizaciones educativas públicas y las segundas son los Comités Cantonales de Deporte y Recreación (CCDR). Según Salazar (2003), dichos Comités nacen en 1966 como agregados a la estructura del Poder Ejecutivo, concretamente de la Dirección General de Educación Física y Deportes. Actualmente, los CCDR son concebidos como entes adscritos a las municipalidades, cuentan con personalidad jurídica instrumental y están sujetos a la estructura del Código municipal, el cual reza en su artículo $\mathrm{N}^{0} 164$, que "los Comités Cantonales de Deportes y Recreación podrán donar implementos, materiales, maquinaria y equipo para dichos programas...a las Juntas de Educación de las escuelas públicas y las Juntas Administrativas de los colegios públicos del respectivo cantón" (Salazar, 2003, p.59).

Este estudio, por tanto, está orientado a respaldar las acciones innovadoras de la Administración de la Educación desde una óptica externa al seno del sistema educativo tradicional, impulsando las relaciones de los centros educativos con su entorno, y darle mayores posibilidades de mejoramiento desde esa óptica, justificando así, la importancia 
Revista Gestión de la Educación, Vol.1, №2, pp.107-143, ISSN 2215-2288, julio-diciembre, 2011

de la gestión conjunta entre las organizaciones educativas públicas y las organizaciones externas que se puedan integrar a colaborar con el mejoramiento de la calidad de la Educación costarricense en general y de la Educación Física en especial.

Consecuentemente, para lograr despejar las interrogantes planteadas, este estudio planteó los siguientes objetivos generales:

1. Analizar la gestión de los centros educativos públicos de secundaria del Circuito 10 de la Dirección Regional de San José, en torno a la reforma del artículo 170 del Código Municipal, en procura del mejoramiento del área de la Educación Física.

2. Diseñar un manual para la administración de la educación que permita mejorar el área de la Educación Física de las organizaciones educativas públicas a partir de la aplicación de la reforma del artículo 170 del Código Municipal.

Por medio de los objetivos específicos se pretende:

1.1 Describir el proceso administrativo ejecutado por las direcciones de los centros educativos del circuito 010 de la Dirección Regional de San José. Específicamente en el área de la Educación Física, según el artículo 170 del Código Municipal.

1.2 Determinar el proceso de coordinación existente entre los centros educativos públicos de secundaria del circuito 10 de la Dirección Regional de San José y la organización municipal del cantón de Montes de Oca.

1.3 Identificar el conocimiento que tienen los actuales administradores de los centros educativos públicos de secundaria con respecto del Código Municipal, los CCDR y las reformas que involucran a las organizaciones educativas con la organización municipal.

1.4 Establecer un análisis comparativo entre las instituciones del estudio, en función del impacto que la gestión ha tenido en el área de la Educación Física en torno a la reforma al artículo 170 del Código Municipal. 
Revista Gestión de la Educación, Vol.1, N², pp.107-143, ISSN 2215-2288, julio-diciembre, 2011

\section{Fundamentación teórica}

\section{Educación: antecedentes y conceptualización}

La educación como proceso debe estar encaminada hacia el futuro mejoramiento del ser humano y como consecuencia, de la sociedad, de manera que el futuro de las generaciones nacientes esté asegurado en el ámbito del desarrollo humano y social, tal

como lo indica Arrién, Bernal, Ooijens, Picón y Thybergin (1996)

La educación es un proceso esencialmente humano, encaminado hacia el futuro y tiene que construirse de manera anticipada con una visión del porvenir. Los estados y sociedades civiles que no se planteen adecuadamente este desafío con todas sus implicaciones, no solo están limitando las alternativas para satisfacer las necesidades educativas de toda la población en el presente, sino que también, se constituyen en un serio obstáculo para el cambio y el desarrollo futuro de las personas, de sus países y del mundo. (p. 39)

Desde esta perspectiva, se presume la obligación del Estado para asegurar la escolarización de los habitantes de la República. Igualmente supone que los procesos adecuados para asegurar dicha escolarización están a cargo del Estado, entre los cuales está incluido la dotación de los recursos materiales y económicos. Esta misma ley propone en su artículo 18 la incorporación de actividades de valor social, ético y estético, entendiendo este último desde un punto de vista físico y relacionado con la actividad física y la salud.

Generalmente, las organizaciones educativas, representadas por sus administradores, no suelen centrar sus esfuerzos solamente a lo interno de la organización, por el contrario, la gestión -como afirma Vargas (2009)- busca que las personas involucradas logren una participación activa en todos los procesos que 
Revista Gestión de la Educación, Vol.1, №2, pp.107-143, ISSN 2215-2288, julio-diciembre, 2011

comprende la gestión del centro, para lograr establecer mecanismos de mejoramiento permanente en sus centros educativos. Esto supone que se brinde a la comunidad estudiantil los recursos necesarios para recibir una educación de calidad desde todos los puntos de vista de las organizaciones educativas, incluyendo la comodidad y la calidad, premisas esenciales de la Ley Fundamental de Educación (1957), la cual indica que todo habitante de la República tiene derecho a la educación y el Estado la obligación de procurar ofrecerla en la forma más amplia y adecuada.

Referente a la educación costarricense, en la década de los 60 se dio un enorme impulso a la educación secundaria y su popularización obligó a pensar en un mejoramiento sustancial en cuanto a las bibliotecas, el otorgamiento de becas, habilitación de comedores, entre otros (Salazar, 2003). Sin embargo, una década después los problemas en este sector de la educación se agravan por la carencia de equipo y apoyos educativos. Precisamente, una de las políticas específicas del Consejo Nacional de Rectores (CONARE) -instancia que integra las universidades públicas en el sistema educativo costarricense para lograr los medios y recursos que faciliten el mejoramiento de la calidad de la educación- es dotar de material didáctico y educativo en general, así como recursos económicos y tecnológicos, adecuados a las necesidades de los distintos grupos de población y a las necesidades institucionales (CONARE, 2006).

El problema de la falta de recursos ha estado presente a lo largo de los últimos años en el sector educación y se extiende conforme crecen los niveles de matrícula; durante el año 2007, estaba matriculado en secundaria, el 71\% de los jóvenes en edades adecuadas para cursar este nivel (Estado de la Educación, 2008).

\section{La administración de la educación y su vinculación con el entorno}

Actualmente, el buen desempeño de un centro educativo puede depender en gran medida del vínculo que exista entre su administración y el entorno en el que esta se encuentra ubicada, al respecto, Arias, Castro, Muñoz y Steller (1995) mencionan lo siguiente:

Número publicado el 20 de diciembre de 2011 URL: http://revistadigital.eae.fcs.ucr.ac.crl 
Revista Gestión de la Educación, Vol.1, №2, pp.107-143, ISSN 2215-2288, julio-diciembre, 2011

Los mecanismos de comunicación que utilizan los administradores de las instituciones educativas son tradicionales y en la actualidad no cumplen con su propósito; por tanto, la deficiente comunicación impide la cooperación, integración y dedicación de padres de familia y dirigentes comunales en la gestión educativa. (p. 120)

El administrador de la educación debe ser consciente de que sus habilidades de negociación, con los integrantes de su organización, con empresas, gobiernos locales y otras organizaciones educativas, son una de sus principales armas para el mejoramiento de esta. "Una política basada en la negociación propicia espacios para el diálogo, respeto, promoción de valores moralmente aceptados (...)" (Garbanzo y Orozco, 2007, p.105). La labor del profesional en administración de la educación se vuelve cada vez más compleja a causa de la naturaleza de sus labores inherentes a su cargo dentro y fuera del centro educativo. A pesar de lo anterior, se hace necesaria la vinculación, cada administrador de la educación debe conocer cada una de las posibilidades con las que cuenta para el mejoramiento de su organización educativa, derivadas de las diferentes organizaciones comunales, gubernamentales, y otras que rodean su zona escolar.

Sobre este tema, Freire (1978) indica que "en un proceso de diálogo entre el educador, el educando y la realidad del entorno está el verdadero proceso de educación que ofrece soluciones posibles y contribuye a la afirmación de los individuos participantes, como hombres mismos" (citado por Castro, 2002, p. 505). Es así, que el personal directivo de la organización es el llamado a propiciar dichos procesos de diálogo, de manera que tanto la organización de proyectos como la puesta en práctica de ideas innovadoras que incluyan a otros sectores de la sociedad, nazcan desde el nivel más operativo del centro educativo, los docentes y los estudiantes. En este sentido, el lograr que se dé una amplia participación del centro educativo con el resto de la comunidad, sean individuos o sean entidades es siempre un reto, tal y como lo expone (Masís, 1995):

Como la educación no es un fin en sí misma, sino que está al servicio de los intereses del individuo y la sociedad, los administradores educativos, deben 
Revista Gestión de la Educación, Vol.1, N², pp.107-143, ISSN 2215-2288, julio-diciembre, 2011

coordinar sus trabajos con los demás sectores, para cumplir con objetivos generales integrales. (p. 51)

De lograrse una buena interacción entre los administradores de la educación y los integrantes de la comunidad, se lograría una serie de beneficios tanto para el centro educativo, como para la calidad de la educación en general. De acuerdo con Castro (2002), estos beneficios se orientan hacia:

1. La solución de problemas concretos que le competen, el mejoramiento de las condiciones materiales como infraestructura, recursos didácticos, etc., y mejores condiciones para la satisfacción de las necesidades educativas de la población estudiantil y sus familias.

2. La coordinación e integración de actividades, esfuerzos y recursos de las diferentes organizaciones e instituciones de la zona, en el logro de los objetivos más importantes de desarrollo educativo y de otro tipo. (p. 510)

Para lograr lo anterior, el administrador de la educación deberá ser consciente de sus capacidades de comunicación, de su desenvolvimiento dentro y fuera del centro educativo, de su sentido de superación constante a favor de su organización y de su capacidad creadora de políticas y reformas pertinentes a las necesidades de la organización educativa en especial y de la administración de la educación en general.

\section{Gestión de la educación}

En el ámbito de la organización educativa, el papel del director adquiere hoy gran relevancia en relación con las funciones que este cumple al frente de la organización y que se complementan con las diferentes capacidades personales que según García et al. (2002) debe tener, tales como: 
Revista Gestión de la Educación, Vol.1, №2, pp.107-143, ISSN 2215-2288, julio-diciembre, 2011

- Temperamento equilibrado

- Tolerancia a la crítica

- Seguridad y capacidad de decisión

- Claridad

- $\quad$ Actitud de reflexión y autocrítica. (p.23)

Un factor importante que debe tener en cuenta el director o directora de una organización educativa son sus capacidades profesionales, que al ser pertinentes contribuyen al logro de resultados óptimos en su labor administrativa. Por lo tanto, deberá considerarlo a la hora de autoevaluar su labor dentro y fuera del centro (Salas y Zamora, 1991).

Al respecto, Garbanzo y Orozco (2010), les dan a las organizaciones educativas un papel de gran relevancia enfocados en la necesidad de contar con un liderazgo personal que lleve a las organizaciones educativas a cumplir con su rol.

Las organizaciones educativas necesitan de un acertado liderazgo para rediseñar modelos de mayor autonomía institucional y gestión moderna de procesos en cada contexto específico, de manera, que permita a las organizaciones no solo el fortalecimiento de sus ofertas académicas, sino los espacios concretos de toma de decisiones que posibilite la combinación adecuada de tiempos, espacios, programas y potencial humano, y con ello cumplir con las expectativas, que hoy día se tienen de las organizaciones educativas. (p. 6)

Existen muchos factores positivos que influyen en el quehacer del personal directivo de la educación sin embargo, no siempre son aprovechados en beneficio de la organización, muchas veces por falta de preparación de estos (as) y otras por la pasividad que muestran a la hora de establecer relaciones con los recursos externos a la organización educativa que dirigen. Williams, citado por García et al. (2002), menciona que es necesario que un gerente o líder tenga las siguientes características: 
Revista Gestión de la Educación, Vol.1, №2, pp.107-143, ISSN 2215-2288, julio-diciembre, 2011

- Habilidad para las buenas relaciones interpersonales

- Buena disposición para comprender a la gente

- Habilidad para comunicarse

- Habilidad para administrar conflictos

- Habilidad para obtener lo mejor de cada uno de los integrantes del grupo

- $\quad$ Capacidad de compromiso. (p.147)

La gestión es un proceso que incluye múltiples y complejas variables, de manera que se requiere vislumbrar nuevos caminos para la construcción de una gestión de la educación capaz de abrir las escuelas al aprendizaje permanente (Vargas, 2009). Estos caminos a los que se hace referencia, no se encuentran demarcados en los textos de la organización educativa, ni en otro tipo de fuente a las que pueda acudir el administrador de la educación, sino, en su propia capacidad y habilidad para construir puentes vinculantes entre su organización y la comunidad que lo rodea, saturada por entidades de tipo político y gubernamental. El administrador de la educación debe ser capaz de preparar el camino de su propio desarrollo dentro de la organización que dirige, pero es claro que no podrá lograrlo si no se apoya en las demás organizaciones.

El concepto estratégico a inspirar una teoría transcendental, es el de la calidad de la educación para todos y todas, considerar lo político, cultural, técnico, pedagógico para la conquista de elevados niveles de calidad de vida humana colectiva (Vargas, 2009).

A pesar de que el grupo administrador de la educación debe contar con la suficiente preparación académica para llevar a cabo en su organización una gestión de calidad, son muchos y muy variados los aspectos que debe manejar para lograrlo, entre estos la gran cantidad de organizaciones que en la actualidad están vinculadas con la institución educativa. Además, una serie de aspectos políticos, culturales y técnicos, a considerar por Sander, citado por Vargas (2009), que deberán ser manejados en forma articulada con su entorno, aprovechando las facilidades que en esos términos le puedan brindar las organizaciones a las que acuda para tal fin, tal y como lo expone García et al. (2002):

Número publicado el 20 de diciembre de 2011 URL: http://revistadigital.eae.fcs.ucr.ac.crl 
Revista Gestión de la Educación, Vol.1, №2, pp.107-143, ISSN 2215-2288, julio-diciembre, 2011

El director, al querer estar en todo, no logra la integración de las diversidades, ni tampoco una adecuada valoración de las capacidades individuales para la conformación de un buen equipo de trabajo, se agota, se agobia y se frustra al desaprovechar los recursos personales presentes en su institución; muchas veces se siente solo y aislado, incomprendido y limitado por un sistema educativo que no lo deja actuar. (p.81)

Todo lo anterior implica que la persona al frente de la organización educativa debe saber qué hacer, cómo hacerlo y conocer con detalle todas sus cualidades administrativas, tales como promover la proyección del centro educativo hacia la comunidad, y de esta a la institución de manera que pueda ponerlas a disposición de sus labores dentro como fuera de la institución que dirige (Meneses, Meoño y Morales, 2005). En este sentido, se debe tomar en cuenta el proceso administrativo que las y los directores de centros educativos desarrollan a lo largo de su gestión al frente de la organización como lo expone Mancebo (1988):

(...) es considerada como una herramienta que la organización puede usar, para incrementar la eficiencia a través de ciertas funciones y principios que los administradores deben desarrollar dentro de los puestos que ocupan en la organización. (p. 43)

Esta definición de proceso administrativo obliga a definir las funciones de la administración, presentadas por Henry Fayol, y que han sido consideradas en todos los campos relacionados con la administración como tal, y que la administración de la educación los adopta acertadamente (Masís, 1995):

Planear: lleva a la organización hacia el establecimiento de sus objetivos. 
Revista Gestión de la Educación, Vol.1, N², pp.107-143, ISSN 2215-2288, julio-diciembre, 2011

Organizar: su función principal es el establecimiento de la estructura de autoridad que deberá seguir la organización.

Coordinar: conjuntar las acciones que las diferentes entidades de la organización desarrollan.

Dirigir: Guiar y motivar para facilitar el logro de los objetivos organizacionales.

Controlar: enfatiza los mecanismos de medición y evaluación como instrumentos que valoran la participación de los diferentes elementos de la organización en el logro de los objetivos.

Importancia de la gestión educativa en los procesos curriculares de la Educación Física

La enseñanza de la Educación Física en la educación secundaria ha estado tradicionalmente orientada por un enfoque deportivo que ha priorizado la medición y la cuantificación de las capacidades físicas de los adolescentes (Pereyra y Vargas, 2006); por ejemplo la estandarización de sus capacidades de saltar (a lo alto y a lo largo), de correr, de hacer abdominales y de resistencia aeróbica (correr sin detenerse durante cierto tiempo, entre otras posibilidades). En muchos casos, las lecciones de Educación Física se han convertido en un espacio de recreo, tanto para las y los estudiantes, como para las y los docentes, precisamente porque se ha manejado con una gestión deficiente o no se le ha brindado el tiempo necesario para su desarrollo efectivo arte de las y los administradores de los centros educativos.

Sin embargo, a pesar del gran auge que ha tenido en los últimos años, la Educación Física no depende solamente de la capacidad de los docentes especialistas en la materia, los cuales dedican todo su esfuerzo y habilidades a la formación física de Número publicado el 20 de diciembre de 2011 URL: http://revistadigital.eae.fcs.ucr.ac.crl 
Revista Gestión de la Educación, Vol.1, N², pp.107-143, ISSN 2215-2288, julio-diciembre, 2011

la población estudiantil, sino también, de los espacios en los que se imparte y de los materiales con los que se cuente para generar actividades.

En primer término, los espacios que se destinen para llevar a cabo las lecciones de Educación Física deben ser amplios, acorde con la cantidad de estudiantes, deben contar con las condiciones adecuadas de limpieza y ventilación. En segundo término, los materiales que se utilicen en la realización de actividades de las lecciones de Educación Física, deben ser suficientes según el número de alumnos que las reciben.

Estos dos componentes (instalaciones y materiales), aunque no son imprescindibles cuando existe la voluntad para enseñar, son de gran valor agregado tanto para el desempeño del docente como para la formación del alumnado, y entre más y mejores materiales e instalaciones se tenga en la organización educativa, aumentarán considerablemente las posibilidades de cumplir con los fines principales de la Educación Física que propone el Ministerio de Educación Pública de Costa Rica, entre los que según Vargas y Orozco (2003), se mencionan los siguientes:

- Propiciar en el educando la identificación con su contexto mediante la observación crítica e interpretación de la realidad de la educación física, del deporte y la recreación que la circunda.

- Contribuir en la formación de un proyecto de vida mediante la valoración de una buena condición física.

- Fomentar en el educando costarricense un adecuado aprovechamiento del tiempo libre, mediante el empleo de actividades físicas, deportivas y recreativas.

- Coadyuvar en la formación integral del individuo, mediante el estímulo de su desarrollo morfológico y motriz.

- Fomentar el desarrollo de habilidades y destrezas psicomotrices.

Precisamente, una educación integral es lo que se busca a través de la enseñanza de la Educación Física, personas físicamente activas, con un amplio sentido de la salud física y mental y sumado a eso, el aporte indirecto al mejoramiento de la población 
Revista Gestión de la Educación, Vol.1, N², pp.107-143, ISSN 2215-2288, julio-diciembre, 2011

estudiantil durante su estadía en el sistema educativo y posteriormente para la vida misma. En este sentido, el aporte del administrador de la educación está orientado principalmente hacia el cumplimiento del primero de los fines de la educación, el cual pretende favorecer el desarrollo armonioso de la salud mental y física del educando. Esta materia deberá dejar de ser la que se utiliza para reuniones de personal, para el recreo solitario del alumnado y profesorado, pero sobre todo, deberá convertirse a través de la gestión, en una forma de crear políticas y reformas educativas que mejoren, desde la perspectiva de la Educación Física y la Administración de la educación, la calidad de la educación que se brinda en las organizaciones educativas.

\section{Relación político-económica de la educación}

Según el Informe de la Cumbre de los Pueblos de América realizada en abril de 1998, el pilar esencial de la educación, la formación de mejores ciudadanos, se pierde en el horizonte, en tanto no se proporcionen los recursos necesarios para impulsar el avance de esta. Las tres acciones de gran relevancia para el cumplimiento de tan importante fin de la educación son aumentar el financiamiento de la educación, elevar la eficiencia en el uso de los recursos públicos que se invierten en educación y asignar recursos en consideración de las distintas realidades y requerimientos económicos y sociales de cada región (Arroyo y Villasuso, 2005).

También se le da un sentido particular al proceso educativo vinculándolo con la política, por cuanto el ser humano como ser social se educa dentro de la sociedad y una de las principales características de esta es la vida política como organización (Dengo, 2005). Igualmente, el aspecto político se pone en contacto con el económico en el momento en que los recursos destinados para la educación se ven afectados por las políticas centralizadas, afectando de manera directa el proceso de mejoramiento de la educación y aumentando sus necesidades desproporcionadamente. 
Revista Gestión de la Educación, Vol.1, N², pp.107-143, ISSN 2215-2288, julio-diciembre, 2011

En toda gestión educativa existe un planteamiento de reforma educativa, y en la mayoría de los casos remite una reforma política, y la dimensión política de la administración de la educación, alude a una reforma educativa. No hay que olvidar que la gran mayoría de los procesos educativos costarricenses están escudados en acciones políticas y que en gran medida cada gobierno es una idea diferente en el plano educativo, tal y como lo exponen Garbanzo y Orozco (2007):

La Administración de la educación, desde un enfoque de competitividad, debe estar en correspondencia con las necesidades de transformación social y económica del país; para lo que se requiere de la articulación de estrategias y políticas educativas en conjunto con las políticas del Estado. (p.99)

En este caso, las y los administradores de la educación son los encargados de velar porque las necesidades de la organización educativa se conviertan en estrategias, que en conjunto con las políticas del estado, conformen el proceso de transformación del centro educativo a favor de la calidad de la educación que se brinda. Así mismo, se plantea que una de las grandes limitaciones que tiene la administración de los centros educativos es la inestabilidad del personal docente y administrativo, inestabilidad que lejos de ayudar al mejoramiento del personal, viene en detrimento de la organización efectiva del centro educativo tal y como lo afirma el CONARE (2006):

Se pierde la continuidad, se pierden los trabajos por construir un proyecto educativo institucional, se puede perder la relación con los padres y madres de familia y con la comunidad en general, se pierde la posibilidad de trabajo en equipo y mucho más. (p.77)

La apreciación anterior da pie para la vinculación de los procesos de administración de la educación con las entidades que de una u otra manera están vinculadas con la política costarricense. De esta manera, se podrá atender las 
Revista Gestión de la Educación, Vol.1, №2, pp.107-143, ISSN 2215-2288, julio-diciembre, 2011

necesidades educacionales de la sociedad, ser realista y factible en términos de los recursos disponibles, ser integral y consciente, ser aplicable a la población, ser coherente con el proyecto político de gobierno y el plan de desarrollo.

\section{Referente metodológico}

La investigación se realizó bajo un enfoque cualitativo exploratorio, dadas las particularidades de la población. Se ejecutaron entrevistas semiestructuradas a los directores y al personal docente de Educación Física de los tres centros educativos participantes del estudio. La información fue sometida a un análisis de contenido temático sustentado en las observaciones de los sujetos consultados. Es exploratoria en el sentido de que no se encontró evidencia de estudios que hayan abordado este tema a fondo (Hernández, Fernández y Baptista, 2006).

Este trabajo de investigación se basa en el aporte al mejoramiento de la Administración de la Educación en la figura de los directores y directoras de los centros educativos, por lo tanto, se toma en cuenta a las y los directamente involucrados en esta disciplina. La población se conformó por las y los directores y profesores de Educación Física de los tres centros educativos de educación secundaria diurnos participantes del estudio. Lo anterior, porque estos centros son los únicos que imparten la Educación Física como una materia más de la oferta curricular de la organización. Igualmente se consultó a un miembro de las Juntas Administrativas y un miembro del Comité Cantonal de Deportes y Recreación de Montes de Oca [CCDRMO]. Dado que la población es pequeña, tres directores, 10 docentes, un miembro de juntas administrativas y un miembro del CCDRMO, se trabajó con la totalidad de los sujetos para la recolección de datos.

Con respecto del instrumento utilizado, se aplicó la validez de criterio, sometiendo los instrumentos al juicio de expertos previo a la aplicación. La información fue recolectada mediante una entrevista semiestructurada compuesta por preguntas cerradas, 
Revista Gestión de la Educación, Vol.1, №2, pp.107-143, ISSN 2215-2288, julio-diciembre, 2011

la cual se aplicó a las y los directores relacionado con la gestión del centro que administran, información acerca del Código Municipal y sus reformas, y con la relación existente entre los centros educativos y los entes municipales.

Se realizó un cuestionario para ser aplicado a las y los profesores de Educación Física de los centros educativos en estudio, acerca del estado actual de sus departamentos de Educación Física en aspectos como material e instalaciones deportivas. Además, se confeccionó una entrevista a un funcionario de la Municipalidad involucrada y otra al presidente o vicepresidente del CCDRMO, y así profundizar en la relación de la Municipalidad con las organizaciones educativas en estudio. De esta manera, el contexto estará más claro desde la perspectiva de todos los involucrados en el proceso de coordinación entre las organizaciones educativas y la organización municipal.

\section{Sistematización y análisis de la información}

El trabajo de campo permitió describir el proceso administrativo ejecutado por las direcciones de los centros educativos en estudio, el proceso de coordinación existente entre las y los directores, y la organización municipal en el cantón de Montes de Oca. También, se identifica el conocimiento que tiene el personal directivo de estas organizaciones con respecto del Código Municipal y los Comités Cantonales de Deporte y Recreación. Todo ello, con el propósito de aportar un mejoramiento en la educación costarricense por medio de la optimización de los procesos de gestión que lleva a cabo el personal directivo de las organizaciones.

Se realiza un acercamiento a las semejanzas y diferencias que se presentan entre los centros educativos del estudio en cuanto a la gestión que se realiza para la mejora de sus instalaciones, materiales, y equipo deportivo y recreativo. Lo anterior, como forma de determinar los procesos administrativos que se establecen entre las organizaciones educativas y su entorno; y por tanto, establecer la importancia que representa un buen manejo, por parte de las y los administradores de la educación, de los recursos externos a 
Revista Gestión de la Educación, Vol.1, N², pp.107-143, ISSN 2215-2288, julio-diciembre, 2011

la organización educativa y canalizarlos hacia el mejoramiento de los procesos de gestión de la Educación Física.

\section{Proceso administrativo seguido por las y los administradores de los centros educativos}

El proceso administrativo es entendido como una herramienta que la organización puede usar para incrementar la eficiencia a través de ciertas funciones y principios que los administradores deben desarrollar dentro de los puestos que ocupan en la organización. Estas funciones se conocen como planificación, organización, coordinación, dirección y control (Mancebo, 1998).

Para poder describir el proceso administrativo que se sigue en las organizaciones educativas que se estudiaron, se debe considerar como indicadores de este proceso las acciones que se derivan de dichas funciones, tales como las estrategias de planificación, las limitaciones en planificación de proyectos de ejecución, el seguimiento curricular y el aprovechamiento del talento humano con que cuenta la organización en sus diferentes áreas. Todo ello como parte de lo que la administración de la educación deberá utilizar al servicio de los centros educativos en conjunción con las demás organizaciones de su entorno. Entre los principales hallazgos del estudio según sus indicadores se encontraron los siguientes:

\section{Estrategias de planificación}

Una planificación eficaz en el esbozo educativo, desde el nivel operativo, debe realizarse para prever resultados desde un ideal previamente determinado y en tres niveles: individual, organizacional y de la sociedad. Dicha planificación debe consistir en la ejecución de estrategias basadas en el diagnóstico institucional, en las prioridades de inversión y en la situación futura deseada (García et al., 2002).

Número publicado el 20 de diciembre de 2011 URL: http://revistadigital.eae.fcs.ucr.ac.crl 
Revista Gestión de la Educación, Vol.1, N², pp.107-143, ISSN 2215-2288, julio-diciembre, 2011

Se evidenció que las y los directores consultados llevan a cabo las acciones necesarias para mejorar en el plano institucional en lo que a deporte y recreación se refiere, tales como planificación, la cual es definida como el planteo y organización de los recursos humanos, materiales y financieros para cumplir con determinados objetivos, supone la coordinación de todos los elementos que participan en un proceso, en aras de un trabajo eficiente. Igualmente promueven una política responsable en la elaboración de planes y proyectos que involucran el proceso de organización, definido como "la tareaactividad por medio de la cual un administrador prevé la coordinación de variables, la estructura y funciones administrativas, las relaciones de autoridad, el empleo y desarrollo de los recursos humanos y materiales, en función de objetivos previamente definidos (Masís, 1995, p. 57).

Estas acciones se desarrollan en el seno de la administración de los centros educativos en coordinación con la administración municipal para favorecer el cumplimiento de los objetivos curriculares de la Educación Física. A pesar de lo anterior, se evidencia la falta de protagonismo que se le otorga, por parte de los directores, al personal, tanto docente como administrativo, en labores de planificación, coordinación y formulación de iniciativas que beneficien al centro educativo desde la perspectiva de los personeros más cercanos a la problemática, las y los educadores físicos.

\section{Aprovechamiento del talento humano}

Las principales respuestas brindadas por los participantes en el estudio evidencian que no se les toma en cuenta para analizar, desde un nivel operativo, las principales necesidades que presenta la organización en cuanto al material e instalaciones deportivas ni para planificar su posible abordaje.

Esta situación llama la atención por cuanto solo un centro educativo toma en cuenta la participación del personal docente y administrativo en labores como la planificación y organización de proyectos dedicados al mejoramiento del área de la Educación Física a través de la renovación de las instalaciones y el material y equipo 
Revista Gestión de la Educación, Vol.1, N², pp.107-143, ISSN 2215-2288, julio-diciembre, 2011

deportivo utilizado para impartir esta materia, contrario a lo que manifiestan la mayoría de docentes con respecto de la misma temática, los cuales mencionan que no se les toma en cuenta para tal efecto (Ver figura 1). Al respecto, una perspectiva lógica desde la Administración de la Educación deja entrever que uno de los principios básicos para asegurar una gestión educativa de calidad, es la capacidad de involucrar a todos y cada uno de los colaboradores de la organización en el cumplimiento de las metas y objetivos de la administración de la educación.

Esta decisión, que deberá tomar el líder de la organización, involucra una gran cantidad de observación que deberá tomar en cuenta antes de hacerlo, y es evidente que la mayoría de las y los administradores de la educación, participantes del estudio, no se toman el tiempo para planificar este tipo de decisiones en busca de la mejor alternativa, lo cual reduce ciertamente la efectividad del proceso administrativo que se desarrolla en la organización. Esta acción es fundamental en el entendido de que el personal del centro educativo se puede convertir en el principal recurso para favorecer el cumplimiento de las metas y objetivos de la organización, tal y como lo menciona García et al. (2002):

Tomando en cuenta el estilo de dirección contingencial, el director o directora deberán tomar en cuenta la madurez de los funcionarios en relación con el deseo de logro, la disposición de aceptar responsabilidades y su habilidad y experiencia relacionada con las tareas que se le asignen. (p. 131)

Es sabido que la calidad de la educación tiene estrecha relación con la plena participación de los individuos en un sistema educativo rodeado de nuevas perspectivas y modelos de desarrollo, por tanto, será imprescindible que las y los administradores de la

educación tomen en cuenta las necesidades del centro educativo, las cuales pueden ser sustentadas por el aporte del personal docente y administrativo de la organización, ligados estrechamente a la función de dirigir, la cual consiste en conducir el talento y esfuerzo de los demás para conseguir resultados. 
Revista Gestión de la Educación, Vol.1, N², pp.107-143, ISSN 2215-2288, julio-diciembre, 2011

Figura 1

Principales acciones administrativas tomadas por los directores, 2010

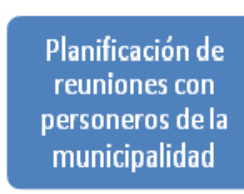

Planificación de

reuniones con

municipalidad

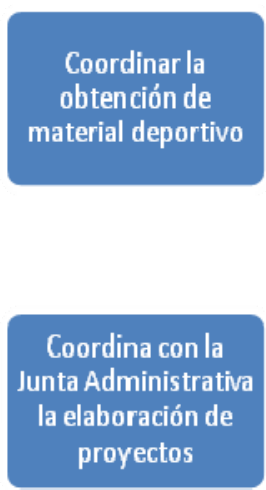

Planificación de

proyectos de

infraestructura

\section{Supervisión del}

cumplimiento delos

objetivos y metas

Fuente: Entrevista estructurada aplicada a los directores (as) de secundaria pública diurna del circuito 10 DRESJ, 2010.

En este sentido, se debe recordar que los que enfrentan el problema de la falta de infraestructura y material deportivo en los centros educativos, son las y los educadores, por lo que darles la oportunidad de participar en este tipo de tareas es indispensable.

El director, al querer estar en todo, no logra la integración de las diversidades, ni tampoco una adecuada valoración de las capacidades individuales para la conformación de un buen equipo de trabajo, se agota, se agobia y se frustra al desaprovechar los recursos personales presentes en su institución; muchas veces se siente solo y aislado, incomprendido y limitado por un sistema educativo que no lo deja actuar. (García et al., 2002, p.81)

El director debe tener presente que una de las grandes cualidades de la administración es la capacidad de delegar en sus compañeros y compañeras de trabajo algunas de las acciones y labores que por sí mismo no puede enfrentar para cumplir con los objetivos de su organización; una buena gestión administrativa, deberá estar acompañada de las principales cualidades del administrador o administradora del centro educativo. Los ejecutivos que no se esfuerzan por adoptar decisiones correctas en cuanto a las personas arriesgan algo más que un bajo rendimiento, ya que "adoptar las Número publicado el 20 de diciembre de 2011 URL: http://revistadigital.eae.fcs.ucr.ac.crl 


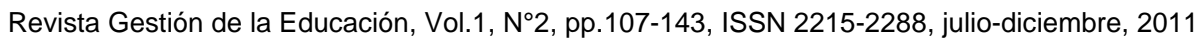

decisiones correctas respecto de la gente es el medio fundamental de controlar bien una organización. Dichas decisiones revelan cuán competente es una administración, cuáles son sus valores y si toma en serio su trabajo" (Drucker, 1987, p. 126).

\section{Proceso de coordinación entre las organizaciones educativas y la organización municipal}

La coordinación, como elemento de la organización, es el componente de la organización encargado de conjuntar las acciones que las diferentes entidades de la organización desarrollan, no solo a lo interno de la institución, sino, en conjunto con las demás organizaciones de la comunidad, tanto públicas como privadas (Mancebo,1998). Para la identificación de estas acciones en las organizaciones educativas del estudio se tomó como referencia cuatro indicadores principales, los cuales son los proyectos realizados en conjunto con la administración municipal, los aportes de las entidades comunales a la organización educativa, la capacidad de gestión de las y los administradores de la educación con las entidades municipales (planificación conjunta) y la evaluación de las actividades concernientes a la organización. Todos ellos, indicadores de los procesos de coordinación que realizan las organizaciones del estudio.

\section{Proyectos realizados en conjunto con la administración municipal}

En relación con el primero de los indicadores mencionados anteriormente, se evidencia, que ninguna de las organizaciones involucradas en el estudio ha realizado planes ni proyectos en conjunto con la administración municipal y mencionan que no

existe ningún proyecto que se haya realizado en beneficio de las organizaciones involucradas. Se debe recordar, que los planes y proyectos en la educación implican su estructuración, ejecución y administración (Masís, 1995). Lo anterior, lleva a pensar que Número publicado el 20 de diciembre de 2011 URL: http://revistadigital.eae.fcs.ucr.ac.crl 
Revista Gestión de la Educación, Vol.1, N², pp.107-143, ISSN 2215-2288, julio-diciembre, 2011

no se está incluyendo, en los planes de la administración de los centros educativos estudiados, el mejoramiento de la infraestructura y el material deportivo y recreativo, lo cual estaría disminuyendo la calidad de las lecciones de Educación Física que se realizan con dichas instalaciones y material.

La administración de la educación a nivel institucional como la que manejan las y los directores de los centros educativos del estudio tiene como objetivo principal el logro de eficiencia en la ejecución del currículo (Masís, 1995). Sin embargo, muchos directores y directoras de centros educativos se olvidan de que para que se cumplan los objetivos curriculares y para mantener la calidad de la educación en su organización, se necesita contar con una serie de cualidades que involucran áreas como la relación con la comunidad, la planificación, la organización y la administración del talento humano, recursos financieros y físicos. Contrario a esto, la atención de las y los administradores de la educación se está desviando de su cauce y se está enfocando más en situaciones administrativas que no necesariamente facilitan el cumplimiento de los objetivos del centro educativo.

\section{Aporte de las entidades comunales a la organización educativa}

En torno al segundo indicador, si se analizan los datos vinculados con el proceso de coordinación que realizan las y los administradores de los centros educativos estudiados, en conjunto con las entidades municipales, se evidencia una inconsistencia entre las acciones que se realizan, y los beneficios que se reciben a favor del centro educativo. Todos los directores afirman que nunca, en el tiempo que han estado al frente del centro educativo, han recibido ayuda por parte del gobierno local de su cantón (Montes de Oca). Sin embargo, esta misma población afirma desconocer el presupuesto que destina el gobierno local a las instituciones educativas. Se podría inferir que el desconocimiento acerca de lo que la organización municipal destina a la educación, le impide al administrador del centro educativo ser competitivo en las estrategias y políticas que se puedan realizar para aprovechar al máximo el aporte municipal. 
Revista Gestión de la Educación, Vol.1, №2, pp.107-143, ISSN 2215-2288, julio-diciembre, 2011

Se debe recordar que, la educación no es un fin en sí misma, sino que está al servicio de los intereses del individuo y la sociedad, por lo tanto las y los administradores de la educación deberán coordinar sus trabajos con los demás sectores, para cumplir con sus objetivos generales. La habilidad de coordinar del director o directora debe reflejarse en las políticas que se desarrollen a favor de la organización, en conjunto con la capacidad de gestión al servicio de los procesos de coordinación con otras entidades, aunque no provengan del nivel central del sistema educativo (ver figura 2).

Generalmente los administradores y administradoras de las demás organizaciones de la comunidad, dedican gran parte de su tiempo a coordinar sus actividades, de tal manera que se dé un desarrollo social integral (Masís, 1995). Para esto se debe conocer con detalle cuales organizaciones rodean el centro educativo y tener claro cuál es la proyección de cada una para identificar la disponibilidad de recursos que se puedan captar por medio de estas.

Figura 2

Organizaciones que los administradores podrían aprovechar como oportunidades para la organización educativa

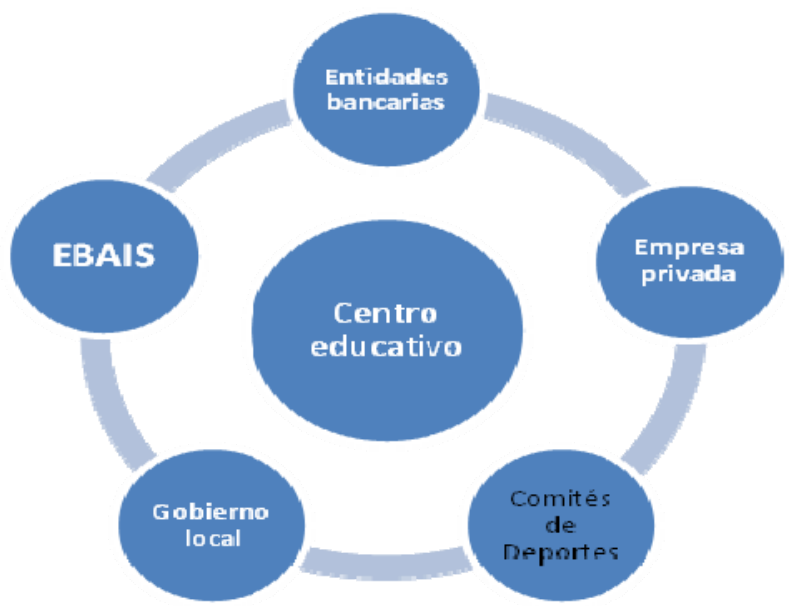

Fuente: Entrevista estructurada aplicada a los directores (as) de secundaria pública diurna del circuito 10 DRESJ, 2010.

Número publicado el 20 de diciembre de 2011 URL: http://revistadigital.eae.fcs.ucr.ac.crl 
Revista Gestión de la Educación, Vol.1, №2, pp.107-143, ISSN 2215-2288, julio-diciembre, 2011

\section{La capacidad de gestión frente a las organizaciones comunales}

Con respecto del tercer indicador, es de gran importancia el tipo de gestión que lleven a cabo las y los administradores de los centros educativos, definida esta por las acciones que se pongan en práctica para favorecer el cumplimiento de los fines y objetivos de la educación. Dicha gestión es entendida según Pozner (2000), citado por Garbanzo y Orozco (2009) como:

Las acciones desarrolladas por los gestores que pilotean espacios organizacionales. Es un saber de síntesis capaz de ligar conocimiento y acción, ética y eficacia, política y administración en procesos que tienden al mejoramiento continuo de las prácticas educativas; a la exploración y explotación de todas las posibilidades y a la innovación permanente como proceso sistemático. (p. 17)

Los resultados del estudio muestran que sí se han gestionado recursos para el mejoramiento de la infraestructura, pero solo en uno de los centros educativos estudiados se han coordinado proyectos de este tipo. Las y los administradores consultados concuerdan en que para que la gestión que realizan en su organización tenga efecto en el mejoramiento de la misma, se deben tomar en cuenta varias estrategias (ver figura 3). Al respecto, Pérez (1981) menciona que las necesidades de más recursos ocasionaría un aumento en el costo de la enseñanza que el país no podría resistir sin realizar un cambio radical con respecto a la recaudación de ingresos para la educación.

Por lo anterior, es que se debe considerar todas las acciones necesarias para que la organización educativa genere sus propios recursos, a partir de una buena gestión por parte de las y los administradores, de manera que se pueda orientar el quehacer educativo con una visión global del entorno (García et al., 2002). 
Revista Gestión de la Educación, Vol.1, №2, pp.107-143, ISSN 2215-2288, julio-diciembre, 2011

Figura 3

Estrategias de gestión llevadas a cabo por los directores y directoras

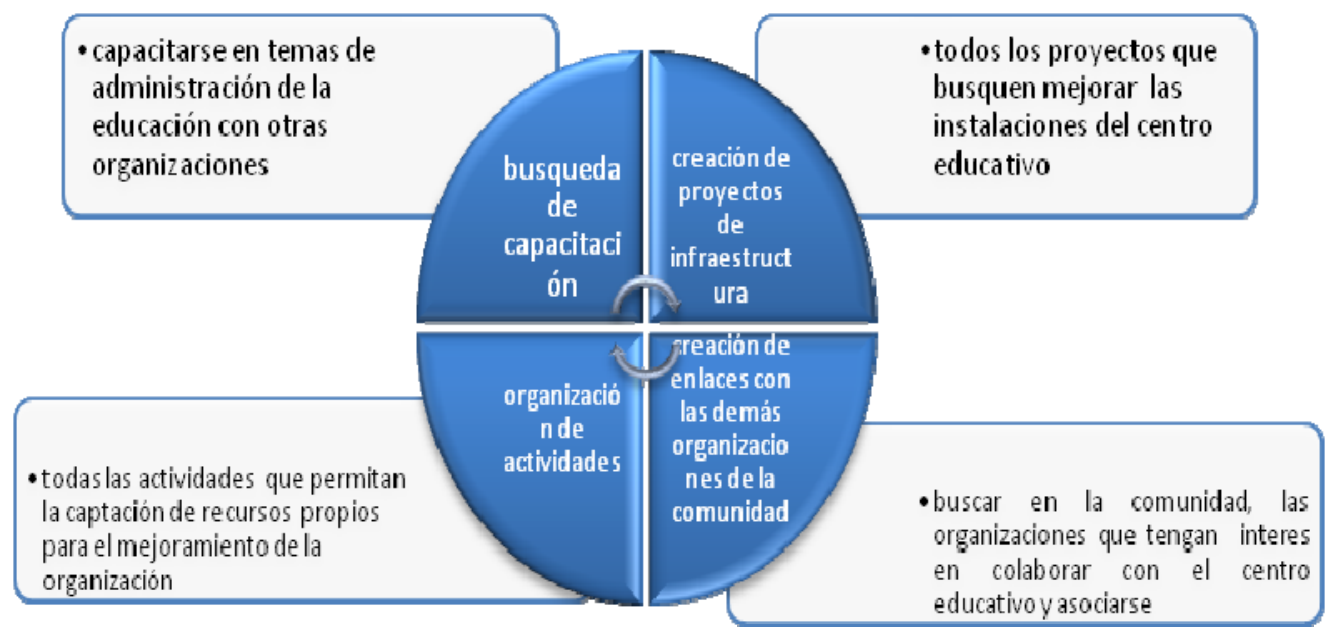

Fuente: Entrevista estructurada aplicada a los directores (as) de secundaria pública diurna del circuito 10 DRESJ, 2010.

\section{Evaluación de las actividades}

En este caso, la evaluación que el director o directora ejerza sobre los procesos de gestión de la organización educativa, es fundamental para el análisis del desarrollo de cada una de las actividades dedicadas al cumplimiento de los objetivos del centro educativo, sean estas internas o externas. La evaluación que deben hacer las y los administradores de la educación supone una forma específica de conocer y de relacionarse con la realidad educativa, "se trata de una praxis transformadora que para incidir en profundidad, precisa activar los resortes culturales, sociales y políticos más relevantes de los contextos en los que actúa" (Andrés, 2000, p.21).

Por tanto, la capacidad evaluadora de los profesionales al frente de los centros educativos en estudio deberá estar presente en todos los procesos de gestión que se den en torno a la organización educativa, desde el nivel más operativo del aula hasta los niveles más altos de decisión. Todo ello, con el fin de mejorar las posibilidades de superación del centro educativo.

Número publicado el 20 de diciembre de 2011 URL: http://revistadigital.eae.fcs.ucr.ac.cr/ 
Revista Gestión de la Educación, Vol.1, N², pp.107-143, ISSN 2215-2288, julio-diciembre, 2011

\section{Conocimiento de los administradores de la educación acerca del Código Municipal y los Comités de Deportes}

Se destaca entre los resultados, el desconocimiento que tienen las y los administradores de la educación al respecto del Código Municipal (Ley N 7794). Lo anterior supone que se desconoce también los artículos de dicho Código mencionados a lo largo del estudio, los cuales son de vital importancia para la o el administrador del centro educativo en el ámbito de la colaboración del gobierno local hacia la organización educativa. Se debe tomar en cuenta que la responsabilidad del administrador de la educación es conocer la legislación y reglamentación necesaria, para desempeñar sus funciones de acuerdo a lo que la ley establece (García et al., 2002).

Particularmente, en lo que se refiere a los procedimientos que se deben utilizar para solicitar ayuda a la municipalidad o al Comité de Deportes por parte de las organizaciones educativas, es clara la respuesta de los sujetos del estudio al mencionar que no los conocen. A pesar de que estos procedimientos no existen, es claro que tampoco se han preocupado por conocer el Código municipal o las reformas que lo modifican en beneficio de las organizaciones educativas, por lo tanto, es de esperar que en ninguna de las organizaciones estudiadas se hayan presentado proyectos que involucren a la organización municipal en el mejoramiento de la calidad de la Educación Física en dichas organizaciones.

Se debe tener en cuenta que las organizaciones educativas necesitan de un acertado liderazgo para rediseñar modelos de mayor autonomía institucional y gestión moderna de procesos en cada contexto específico (Garbanzo y Orozco, 2009); sin embargo, ese liderazgo no se evidencia según los resultados observados. En este mismo sentido, la importancia que los administradores le dan al vínculo existente entre su conocimiento del Código Municipal y su desempeño como directores (as), parece estar dividida entre las organizaciones educativas involucradas en el estudio, mientras para unos su desempeño se ve afectado por el desconocimiento del Código Municipal, para otros eso no representa ningún problema en su gestión, es decir, solo uno afirma que conocer acerca del Código Municipal le traerá beneficios al centro educativo. 
Revista Gestión de la Educación, Vol.1, N², pp.107-143, ISSN 2215-2288, julio-diciembre, 2011

\section{Limitaciones que supone el desconocimiento}

Este análisis lleva a persuadir a los administradores y las administradoras de la educación en cuanto a las consecuencias que eventualmente puede traer a las organizaciones educativas la falta de un conocimiento amplio acerca de los temas adyacentes al centro educativo, consecuencias como la falta de apoyo de las organizaciones comunales, incapacidad para gestionar proyectos en coordinación con el gobierno local, entre otras, y consecuentemente, se pierde la oportunidad para mejorar la calidad de la Educación Física con más y mejor infraestructura y material deportivo (ver figura 4).

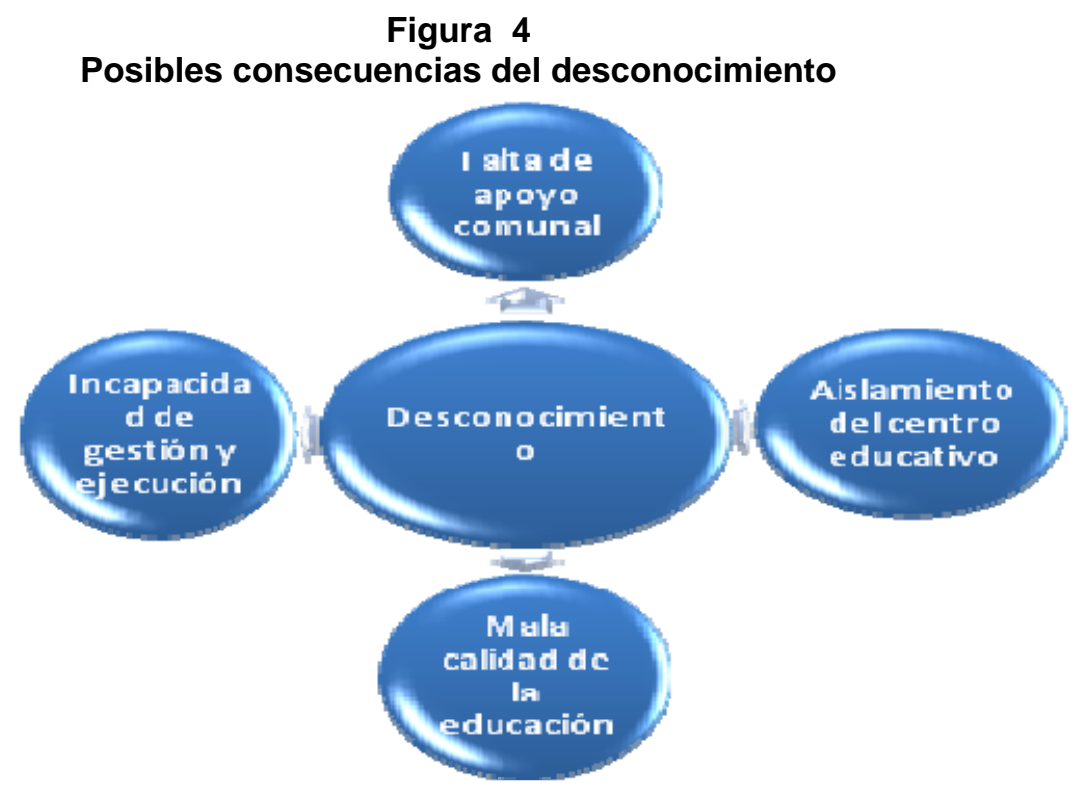

Fuente: Entrevista estructurada aplicada a los directores (as) de secundaria pública diurna del circuito 10 DRESJ, 2010.

Por lo tanto, para minimizar la influencia del deficiente dominio que tienen las y los administradores de la educación acerca del tema analizado en este apartado, es esencial que ellos mismos tengan la capacidad de planear el futuro de su organización, de el contexto completo a su alrededor y no esperar a que las debilidades y amenazas de su Número publicado el 20 de diciembre de 2011 URL: http://revistadigital.eae.fcs.ucr.ac.crl 
Revista Gestión de la Educación, Vol.1, N², pp.107-143, ISSN 2215-2288, julio-diciembre, 2011

organización se conviertan en realidades irreversibles (García et al., 2002). Deberán conocer la mayor cantidad posible de alternativas para procurar el mejoramiento de la organización que dirigen, incluyendo las leyes y reformas que no afectan directamente a la educación.

\section{Relación entre las instituciones estudiadas}

No hay duda de que tanto los administradores como los docentes concuerdan en que la infraestructura deportiva del centro educativo y el material y equipo deportivo ayuda al cumplimiento de los objetivos curriculares de la Educación Física en especial y de la calidad de la educación en general de la organización, lo cual deja entrever que están conscientes de la importancia de su utilización en los planes y programas pedagógicos que se lleven a cabo en el centro educativo.

Cabe destacar, que las poblaciones de los centros educativos estudiados son muy similares, sin embargo las instalaciones y material deportivo con que se cuenta son muy diferentes entre cada organización. Existe una clara diferencia en la capacidad de las instalaciones para albergar a la población estudiantil y una evidente falta de espacio entre una organización y otra.

Un hallazgo importante, es el hecho de que la mitad de los docentes consultados concuerdan en que la cantidad de material deportivo que tiene el centro educativo es insuficiente para la cantidad de estudiantes que reciben Educación Física, y a pesar de que las tres organizaciones estudiadas cuentan con una extensión grande de terreno, las instalaciones deportivas son muy reducidas. Cerca de la mitad de la población de los docentes consultados creen que con el material existente se cumple con los requerimientos mínimos del currículo de la Educación Física.

La totalidad de los docentes participantes del estudio coinciden en que las instalaciones deportivas del centro educativo cumplen con los requerimientos mínimos para el cumplimiento de los objetivos curriculares de la Educación Física, sin embargo, la 
Revista Gestión de la Educación, Vol.1, N², pp.107-143, ISSN 2215-2288, julio-diciembre, 2011

misma población está de acuerdo en que es necesaria una renovación urgente tanto del material como de las instalaciones deportivas en el centro educativo.

Esta información revela que, aunque se cuenta con infraestructura deportiva, esta no reúne las condiciones necesarias para el desarrollo de las lecciones de Educación Física, precisamente porque es infraestructura construida hace ya muchos años y pensada para grupos de 25 a 30 estudiantes, actualmente en las mismas instalaciones se trabaja con 45 o más estudiantes por lección, lo cual reduce considerablemente la eficiencia con que se ejecutan los contenidos curriculares de la Educación Física. Existe también la afirmación de que la planta física y el material no lo es todo, tal y como apunta Masís (1995)

(...) más que la planta física y los recursos didácticos, es la actitud de los docentes y los administradores la que hace posible cierto grado de eficiencia en la enseñanza. Es el amor a los educandos, las buenas relaciones humanas entre compañeros, la disciplina de trabajo y el sentido común lo que convierte un galerón en un espacio apto para la enseñanza. (р. 233)

La información obtenida con el estudio, de cierta forma, justifica la percepción que tienen los personeros de las Juntas Administrativas, los cuales mencionan en las entrevistas realizadas, que "es muy difícil adquirir materiales en general y ejecutar proyectos de infraestructura por la cantidad tan grande de papeleo que hay que realizar ante el Ministerio de Educación Pública, entre estos papeleos se debe contratar profesionales en ingeniería y arquitectura y los costos son muy elevados".

En el caso de los materiales y equipo deportivo con que se cuenta, solo en uno de los casos es suficiente para que las lecciones de Educación Física se desarrollen acorde con el planeamiento de los docentes. En la medida de lo posible, se tiene el material para atender a un aproximado de 35 estudiantes por lección, lo que representa una aplicación fluida de los fundamentos a enseñar con el material. Sin embargo, en las demás organizaciones estudiadas, el material es muy escaso y no alcanza para abarcar a más de la mitad de los estudiantes por lección. Según la totalidad de los docentes consultados, 
Revista Gestión de la Educación, Vol.1, N², pp.107-143, ISSN 2215-2288, julio-diciembre, 2011

"la cantidad de material con el que cuentan se tiene que repartir entre todos los profesores y se reduce considerablemente cada año por pérdidas o daños".

\section{Estado y pertinencia de las instalaciones y material}

La importancia de la planta física y el material deportivo de una organización educativa, radica en que esta sea apropiada para su utilización, y que permita el buen desarrollo de los objetivos de la Educación Física, abarcando la totalidad de la población estudiantil. Existen variables que determinan la organización de la planta física y que el administrador o administradora de la educación deberá interrelacionar de tal forma que se logre la eficiencia, tanto del proceso como del producto educativo (Masís, 1995), esta información se ilustra en la siguiente figura:

Figura 5

Variables que determinan la organización de la planta física y el material deportivo de la organización educativa

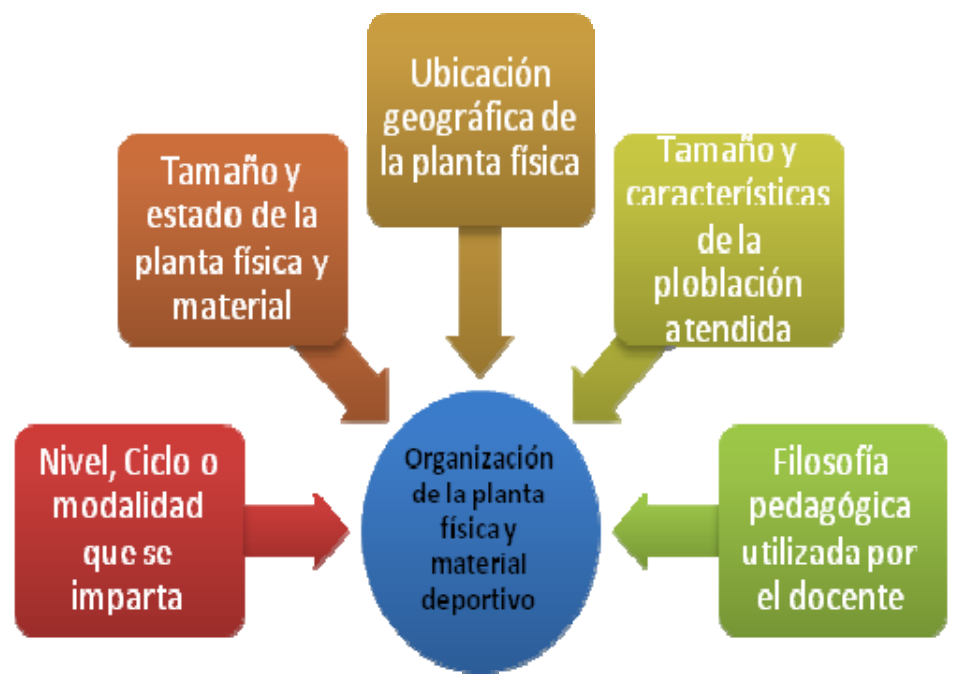

Fuente: Adaptado de Masis (1995).

Número publicado el 20 de diciembre de 2011 URL: http://revistadigital.eae.fcs.ucr.ac.crl 
Revista Gestión de la Educación, Vol.1, N², pp.107-143, ISSN 2215-2288, julio-diciembre, 2011

En el caso de las organizaciones estudiadas, se evidenció que con la calidad y desempeño de los docentes en Educación Física, se logran la mayoría de los objetivos curriculares de esta disciplina, a pesar de que la cantidad de instalaciones y material deportivo no es el apropiado para impartir las lecciones tal y como se planifican por parte del docente. En la mayoría de los casos, los docentes consultados están de acuerdo en que la infraestructura con la que cuentan no les permite desarrollar al máximo su planeamiento diario, principalmente por la cantidad tan grande de estudiantes que se atienden por lección. Igualmente, la mayoría de los docentes afirma cumplir con los requerimientos curriculares del Ministerio de Educación a pesar de esta situación.

Con respecto del material deportivo, se evidenció la necesidad de renovarlo casi en su totalidad. Por la escasa cantidad de material se hace necesaria la utilización del que se encuentra en condiciones no aptas para la enseñanza. Esto desemboca en la negativa de las y los estudiantes de no utilizarlo, prefieren no realizar las actividades planificadas, ni atender indicaciones y se crea un ambiente de rechazo hacia las lecciones de Educación Física.

\section{Consideraciones finales}

Se debe recordar que en el caso particular de la Educación Física, el aporte a la calidad de la educación, se da por medio del mejoramiento de las capacidades físicas que desarrolle cada estudiante, así como las destrezas y habilidades que se adquieran a lo largo de su vida como estudiante y finalmente con la formación de individuos integrales capaces de desenvolverse en una sociedad que cada día solicita más y mejores cualidades físicas y mentales de los ciudadanos activos salientes del sistema educativo.

Es por ello que al finalizar esta discusión, se pretende involucrar a las y los administradores de la educación en el manejo efectivo de los procedimientos que se plantean para que, por medio del mejoramiento de la planta física y el mejoramiento de la cantidad y la calidad de los materiales y equipos deportivos gestionados a través de la administración municipal se logre el fortalecimiento de la administración de la educación y 
Revista Gestión de la Educación, Vol.1, N², pp.107-143, ISSN 2215-2288, julio-diciembre, 2011

por consiguiente el aumento fundamental en la calidad de la educación y del sistema educativo costarricense en toda su estructura.

Es importante que los administradores de la educación dejen de ver la Educación Física como una materia especial, y la reconozcan como una materia complementaria dentro del plan de desarrollo curricular tal y como lo ha establecido el Ministerio de Educación Pública. Lo anterior, no como forma de realce hacia la Educación Física, sino como una forma de recuperar el lugar que esta se merece como coadyuvante en el logro de los objetivos de la organización.

Las y los administradores de la educación deberán fomentar los intercambios entre la organización educativa y las demás organizaciones que rodean el centro educativo en procura de poner en práctica las propuestas que en la cotidianidad surgen a lo interno de la organización y que con el apoyo de las entidades comunales como los gobiernos locales, tendrán una mayor y mejor puesta en práctica para beneficio de la educación en general.

\section{Figura 6}

Cualidades de los y las administradoras de la educación frente a los desafíos actuales

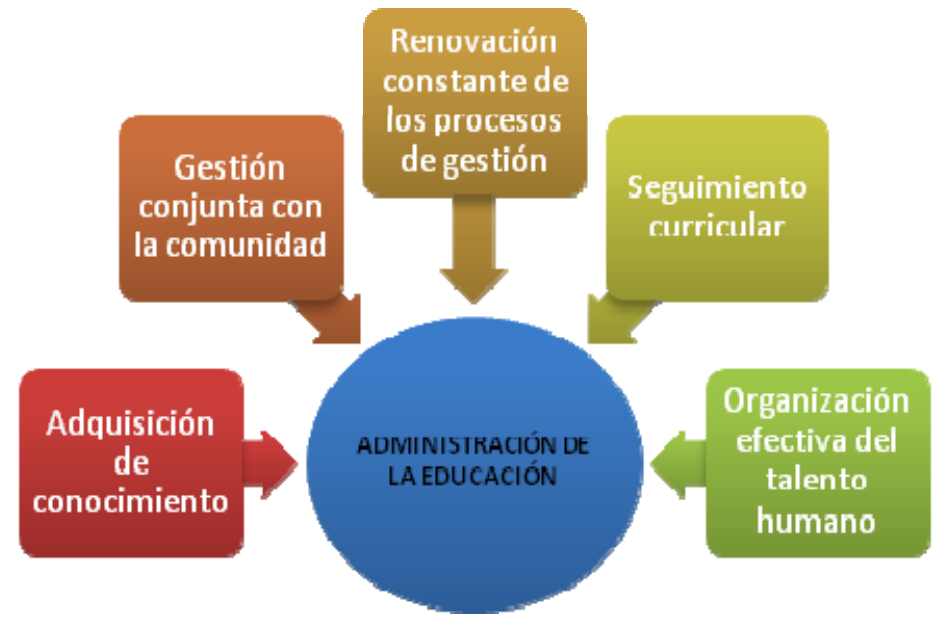

Fuente: Elaboración propia (2010).

Número publicado el 20 de diciembre de 2011 URL: http://revistadigital.eae.fcs.ucr.ac.crl 
Revista Gestión de la Educación, Vol.1, N², pp.107-143, ISSN 2215-2288, julio-diciembre, 2011

Se debe prestar especial atención a las cualidades de las y los administradores en el campo de la gestión, de manera que los procesos de planificación, organización, coordinación, dirección y control sean renovados constantemente y destinados al mejoramiento de los procesos educativos, impulsando así, la sensibilización de la administración de la educación hacia los cambios educativos propuestos en defensa de la educación en general y de la Educación Física en especial.

La administración de la educación debe tomar un rumbo estratégico, orientado hacia la consecución de los objetivos y metas planteadas desde el nivel central hasta el nivel más operativo, con una proyección a futuro que brinde la oportunidad de mejorar los procedimientos de gestión utilizados por las y los administradores de la educación costarricense. Para ello, los líderes de las organizaciones educativas deben tener clara la complejidad de la organización y de todos los procesos que esta conlleva, de manera que pueda establecer adecuadamente las posibilidades reales de desarrollo institucional con la ayuda tanto de los factores internos como de los externos.

Las relaciones interpersonales entre las y los administradores de la educación y las diferentes entidades que rodean su centro educativo son motivo de reflexión para que se den de una mejor forma los procesos de coordinación entre los dos entes, de manera que a través de esa coordinación surjan las claves que le darán el sustento a la ejecución de planes y proyectos en conjunto que le den mayor credibilidad a la administración de la educación y sus procesos de gestión en beneficio de las organizaciones educativas y sus objetivos. Las y los directores de los centros educativos, no parecen ser conscientes de que es necesaria la trascendencia de sus labores dentro de los límites, hacia el exterior del centro educativo, en busca de alianzas estratégicas que le den a la organización un panorama más amplio en su gama de posibilidades de crecimiento. 


\section{Referencias bibliográficas}

Andrés, J. M. (2000). La evaluación educativa, su práctica y otras metáforas. Barcelona: Editorial Horsori.

Arias, J.; Castro, B.; Muñoz, J. y Steller, J. (1995). Mecanismos que utilizan los administradores de los colegios académicos diurnos oficiales. Tesis para optar por el grado de Licenciatura en Ciencias de la Educación con mención en Administración Educativa. Universidad de Costa Rica, San Ramón, Alajuela.

Arrién, J; Bernal, J; Ooijens, J; Picón, C y Thybergin, A. (1996). Calidad de la educación en el istmo centroamericano. Oficina de la UNESCO para Centroamérica y Panamá. San José, Costa Rica: Editorial NORMA.

Arroyo, M.V. y Villasuso, E. (2005). Dimensiones de la educación en Costa Rica. San José, Costa Rica: Fundación Friederich Ebert.

Consejo Nacional de Rectores [CONARE]. (2006). Hacia un modelo educativo para elevar localidad de la educación costarricense. Una propuesta de políticas, estrategias y acciones. San José, Costa Rica. Recuperado de http://www.conare.ac.cr/docs/mod educ 2006/CONARE 00.pdf

C.R. (1957). Ley Fundamental de Educación. Recuperado de http://www.apse.or.cr/webapse/legdoc/leg02.htm

C.R. (2003). Código municipal y legislación conexa. Ley 7794. San José, Costa Rica: Editec Editores.

Dengo, M. (1995). Educación costarricense. San José, Costa Rica: EUNED.

Garbanzo, G. y Orozco, V. (2007). Desafíos del sistema educativo costarricense: un nuevo paradigma de la Administración de la Educación. Revista Educación, 31(2), 95-110. Universidad de Costa Rica, San José, Costa Rica. Recuperado de http://www.latindex.ucr.ac.cr/educacion-31-2/06-GARBANZO.pdf

Garbanzo, G. y Orozco, V. (2010). Liderazgo para una gestión moderna de procesos educativos. Revista Educación, 34(1), 15-29. Universidad de Costa Rica, San José, Costa

Rica.

Recuperado

de http://redalyc.uaemex.mx/redalyc/pdf/440/44013961001.pdf 
Revista Gestión de la Educación, Vol.1, №2, pp.107-143, ISSN 2215-2288, julio-diciembre, 2011

García, N; Rojas M. y Campos N. (2002). La administración escolar para el cambio y el mejoramiento de las instituciones educativas. San José, Costa Rica: Editorial UCR.

Hernández, R.; Fernández, C. y Baptista, P. (2006). Metodología de la investigación. México: McGraw-Hill Interamericana.

Mancebo del Castillo, J.M. (1998). El administrador y su entorno dentro de la administración. México, D.F.: Editorial Limusa.

Masís, C. (1995). Administración de instituciones o procesos educativos. San José, Costa Rica: Editorial EUNED.

Meneses, G.; Meoño, H y Morales, L. (2005). Requerimientos legales del administrador educativo para el ejercicio óptimo de su labor en el circuito 04 de la Dirección Regional de Cartago. Tesis para optar por el grado de Licenciatura en ciencias de la Educación con mención en Administración Educativa. Universidad de Costa Rica, San José, Costa Rica.

Organización de las Naciones Unidas [ONU]. (2009). Objetivos de desarrollo del milenio. Informe 2009. Recuperado de http://www.cinu.org.mx/ODM3

Pereyra, A. y Vargas, S. (2006). Reforma de la Educación Secundaria. Fundamentación Curricular. Educación Física. México: Secretaría de Educación Pública. Recuperado de:

http://www.reformasecundaria.sep.gob.mx/doc/FUNDAMENTACIONES/FISICA.pdf

Pérez, H. (1981). Educación y Desarrollo. San José, Costa Rica: Editorial Costa Rica.

Pila, A. (2002). Evaluación de la Educación Física. San José, Costa Rica: Pila Teleña.

Programa Estado de la Nación. (2008). Segundo Informe Estado de la Educación. San José, Costa Rica: Autor.

Salazar, J. (2003). Historia de la educación costarricense. San José, Costa Rica: EUNED.

Segunda Cumbre de las Américas. (1998). Informe de la Cumbre de los Pueblos de América. Declaración de principios. Santiago, Chile.

Vargas, P. y Orozco, R. (2003). La importancia de la Educación Física en el currículo escolar. Revista Inter sedes, 5 (7), Universidad de Costa Rica, San José, Costa Rica: Editorial UCR. 